\title{
Supporting Information: Degrafting of Polymer Brushes by Exposure to Humid Air
}

\author{
Maria Brió Pérez, Marco Cirelli, and Sissi de Beer* \\ Materials Science and Technology of Polymers, MESA+ Institute for Nanotechnology, \\ University of Twente, P.O. Box 217, 7500 AE Enschede, the Netherlands \\ E-mail: s.j.a.debeer@utwente.nl \\ Phone: +31534893170
}




\section{List of acronyms}

RH Relative humidity

ATRP Atom Transfer Radical Polymerization

BiBB $\quad \alpha$-bromoisobutyryl bromide

EBiB ethyl $\alpha$-bromoisobutyrate

APTES (3-aminopropyl)triethoxysilane

PGMA poly(glycidyl metacrylate)

bipy 2,2'-bipyridyl

TEA triethylamine

HEMA 2-hydroxyethyl methacrylate

PHEMA poly(2-hydroxyethyl methacrylate)

MMA methyl methacrylate

PMMA poly(methyl methacrylate)

SPMAK 3-sulfopropyl meth-acrylate potassium

PSPMA poly(3-sulfopropyl meth-acrylate potassium)

MPC 2-methacryloyloxyethyl phosphorylcholine

PMPC poly(2-methacryloyloxyethyl phosphorylcholine)

DMF dimethyl formamide

FTIR Fourier Transfer InfraRed spectroscopy

AFM Atomic Force Microscopy

${ }^{1}$ H-NMR Proton Nuclear Magnetic Resonance 


\section{Experimental details}

\subsection{Materials}

Silicon wafers $(100.0 \pm 0.5 \mathrm{~mm}$ diameter and $525 \pm 25 \mu \mathrm{m}$ thickness, boron-doped with (100) orientation, 5-10 $\Omega \mathrm{cm}$, Okmetic) were cut in $1 \mathrm{x} 1 \mathrm{~cm}$ squares and were used as surface for polymer brush coatings. Each polymer brush was grown in three different substrates and those were used for long-term stability analysis. The same type of substrate was used for AFM measurements. Hydroxyethylmethacrylate (HEMA, Aldrich, 98\%) and methyl methacrylate (MMA, Aldrich, 99\%) contained inhibitors, which were removed by passing the monomer solution through an aluminum oxide column. Copper (I) bromide ( $\mathrm{CuBr}$, Aldrich, 98\%) was cleaned with acetic acid (analytical standard, Aldrich) and washed with ethanol (EtOH, Merck, AR). 2-Methacryloyloxyethyl phosphorylcholine (MPC, Aldrich, 99\%), 3-Sulfopropyl methacrylate potassium salt (SPMAK, Aldrich, 98\%), copper (II) bromide $\left(\mathrm{CuBr}_{2}\right.$, Aldrich, 99\%), (3-aminopropyl)triethoxysilane (APTES, Aldrich, 99\%), triethylamine (TEA, Aldrich, $\geq 99 \%$ ), toluene (Aldrich, dried), $\alpha$-bromoisobutyryl bromide (BiBB, Aldrich, 98\%), ethyl $\alpha$-bromoisobutyrate (EBiB, Aldrich, 98\%) and 2,2'-Bipyridyl (Bipy, Aldrich, $\geq 99 \%$ ), dimethyl sulfoxide-d6 (DMSO-d 6 , $99.96 \%$ atom D, Aldrich), chloroform $\left(\mathrm{CDCl}_{3}, 99.8\right.$ atom\% D, Aldrich) and deuterium oxide $\left(\mathrm{D}_{2} \mathrm{O}, 99.9\right.$ atom\% $\mathrm{D}$, Aldrich) were purchased and used as received without purification. Hydrogen peroxide $\left(\mathrm{H}_{2} \mathrm{O}_{2}\right.$, Merck, $30 \%$ ) was used as received. Methanol (AR), acetone (AR) and sulfuric acid (95-97\%) were purchased from Biosolve and used as received. MilliQ water was purified from a MilliQ Advange A10 purification system (Millipore, Billerica, Ma, USA). Saturated salt solutions were prepared with sodium chloride $(\mathrm{NaCl}, \geq 99.0 \%$, Aldrich $)$, potassium sulfate $\left(\mathrm{K}_{2} \mathrm{SO}_{4}\right.$, $\geq 99 \%$, Aldrich), lithium chloride ( $\mathrm{LiCl}, \geq 99 \%$, Aldrich) and magnesium chloride $\left(\mathrm{MgCl}_{2}\right.$, $\geq 98 \%$, Aldrich). Relative humidity control inside closed chambers was performed with a SEK-Environmental sensing evaluation kit (Sensirion), consisting of a SEK-SensorBridge and a SEK-SCC30-DB-Sensor humidity sensor. The software SEK-ControlCenter viewer 
was used for the display and log of the sensor signals for multiple sensors connected to the SEK-SensorBridge.

\subsection{Substrate pre-functionalization}

Prior to the synthesis of polymer brushes on silicon surfaces, two functionalization steps were performed on the silicon substrates, as shown in the main text in Figure 1. First, the substrates were cleaned with Piranha solution $\left(\mathrm{H}_{2} \mathrm{SO}_{4}: \mathrm{H}_{2} \mathrm{O}_{2}=3: 1, \mathrm{v} / \mathrm{v}\right)$ for the removal of organic contaminants and the hydroxylation of the surface (addition of $\mathrm{OH}$ groups), which renders it hydrophilic. Piranha cleaning was performed for 30 minutes at room temperature and the samples were subsequently rinsed with water, ethanol and dried with nitrogen stream.

Next, (3-aminopropyl)triethyoxysilane (APTES) anchoring layers were anchored to the clean silicon substrate, with the purpose of studying their effect in the long-term stability of polymer brushes in humid air. Chemical vapor deposition was the selected method, established in previous literature. ${ }^{1}$ The clean substrates were stored in a desiccator, together with a small plate containing approximately $0.1 \mathrm{~mL}$ of APTES solution. Vacuum was first applied for 15 minutes, followed by the closing of the desiccator chamber. This way, the vapor deposition of APTES was allowed overnight. After that, the samples were rinsed with water, ethanol and dried with nitrogen stream.

After that, $\alpha$-bromoisobutyryl bromide (BiBB) was grafted, following previous methodology. ${ }^{2}$ The substrates were placed in a sample holder in order to avoid sample damage and salt adhesion during the course of the reaction. The samples were placed inside a beaker with a solution of cold toluene and triethylamine (TEA). $\alpha$-bromoisobutyryl bromide (BiBB), our selected initiator, was added drop-wise to the solution at constant stirring. The reaction was carried out for one hour, following the ratios Toluene:TEA:BiBB=300:1:1 v/v.

After the deposition was performed, the substrates were rinsed with ethanol, water and dried with nitrogen stream. 


\subsection{Synthesis of polymer brushes}

We used surface-initiated atom transfer radical polymerization (SI-ATRP) in order to grow four different brushes from silicon surfaces, following well-established previous recipes together with in-house ones. ${ }^{1-3}$ The grown polymer brushes were: poly(methyl methacrylate) (PMMA), poly(2-hydroxyethyl methacrylate) (PHEMA), poly(2-methacryloyloxyethyl phosphorylcholine) (PMPC) and poly(3-sulfopropyl methacrylate) (PSPMA).

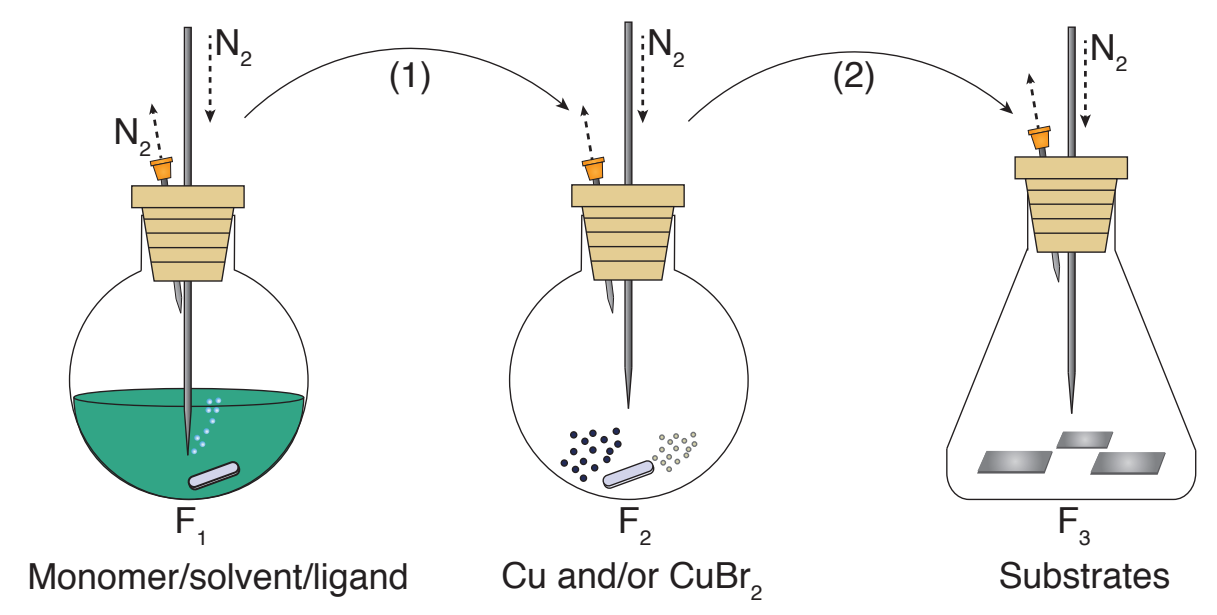

Figure S1: SI-ATRP procedure for polymer brush growth.

In a round bottom flask sealed with a septum ( $\mathrm{F}_{1}$ in Figure $\left.\mathrm{S} 1\right)$ a solution containing the selected monomer, ligand and solvent(s) was purged with nitrogen and mixed at constant stirring (1 minute degassing $/ \mathrm{ml}$ solution). In a second round bottom flask ( $\mathrm{F}_{2}$ in Figure $\mathrm{S} 1$ ) the copper powder(s) were degassed for at least 30 minutes. Next, the solution of $F_{1}$ is added to the second flask $\mathrm{F}_{2}$ with a purged syringe. ${ }^{\mathrm{i}}$ The solution was stirred and degassed until all the copper species were dissolved. After that, the solution is syringed over the substrates via a purged needle and the polymerization is allowed to proceed for a certain period of time. ${ }^{\text {ii }}$ By removing the septum and allowing oxygen to enter the flask, the polymerization

\footnotetext{
${ }^{i}$ The syringe used for transferring solvents has to be purged with nitrogen three times to avoid oxygen entering the inert flask.

${ }^{\text {ii }}$ Consider the over-pressure; when subtracting a liquid from a sealed flask, remove the outlet from the septum. When adding additional volume to a sealed flask, keep the outlet in, or explosions may occur due to over-pressure. The outlets should also be removed from the septum of all the reactions flasks connected to the nitrogen lines when subtracting liquids, in order to avoid suction of the mixtures via the needles into the line due to pressure changes.
} 
process could be stopped. Finally, the functionalized samples with polymer brushes were rinsed with water, ethanol and dried with nitrogen stream.

The reactants mol ratio for the synthesis of all the polymer brushes studied in this work were:

- For PMMA brushes: MMA:bipy: $\mathrm{CuBr}=100: 2: 1$, in a 1:4 v/v water-methanol solution at $25^{\circ} \mathrm{C}$. Polymerization time of two hours.

- For PHEMA brushes: HEMA:bipy:CuBr: $\mathrm{CuBr}_{2}=100: 5: 1: 0.1$, in water at $25^{\circ} \mathrm{C}$. Polymerization time of two hours.

- For PSPMA brushes: SPMAK:bipy:CuBr: $\mathrm{CuBr}_{2}=110: 5: 1: 0.1$, in a $1: 2 \mathrm{v} / \mathrm{v}$ watermethanol solution at $25^{\circ} \mathrm{C}$. Polymerization time of two hours.

- For PMPC brushes: MPC:bipy:CuBr: $\mathrm{CuBr}_{2}=60: 3: 1: 0.3$, in a 1:1 v/v water-methanol solution at $25^{\circ} \mathrm{C}$. Polymerization time of one hour. 


\subsection{Synthesis of free polymers in solution}

Polymer brushes grown simultaneously in solution from a sacrificial initiator (ethyl $\alpha$ bromoisobutyrate, EBiB) and from surfaces were synthesised with the purpose of obtaining an estimate of the brush molecular weight $\left(\mathrm{M}_{\mathrm{n}}\right)$.

When growing polymer brushes with a moderately high grafting density, the estimation

of the molecular weight of the thin film by means of polymerization in solution can be considered valid. Polymerizations in solution were conducted using the following mol ratios:

- For PMMA brushes: MMA:bipy:CuBr:EBiB=100:2:1:1, in a 1:4 v/v water-methanol solution at $25^{\circ} \mathrm{C}$. Deuterated chloroform was the solvent used for ${ }^{1} \mathrm{H}-\mathrm{NMR}$ experiments.

- For PHEMA brushes: HEMA:bipy:CuBr: $\mathrm{CuBr}_{2}: \mathrm{EBiB}=100: 5: 1: 0.1: 0.2$, in water at $25^{\circ} \mathrm{C}$. DMSO- $\mathrm{d}_{6}$ was used as solvent for ${ }^{1} \mathrm{H}-\mathrm{NMR}$ experiments.

- For PSPMA brushes: SPMAK:bipy:CuBr: $\mathrm{CuBr}_{2}: \mathrm{EBiB}=110: 5: 1: 0.1: 0.2$, in a 1:2 v/v water-methanol solution at $25^{\circ} \mathrm{C} . \mathrm{D}_{2} \mathrm{O}$ was used as solvent for ${ }^{1} \mathrm{H}-\mathrm{NMR}$ experiments.

- For PMPC brushes: MPC:bipy: $\mathrm{CuBr}: \mathrm{CuBr}_{2}: \mathrm{EBiB}=60: 3: 1: 0.3: 1$, in a 1:1 v/v watermethanol solution at $25^{\circ} \mathrm{C} . \mathrm{D}_{2} \mathrm{O}$ was the solvent used for ${ }^{1} \mathrm{H}-\mathrm{NMR}$ experiments.

Dimethyl formamide (DMF) was degassed and used as an internal standard for all reactions. A small volume $(0.1 \mathrm{~mL})$ was added to the reaction flask right before the start of each polymerization. 


\subsection{Relative humidity control in closed chambers}

Four different saturated salt solutions were prepared, adapting previous procedures. ${ }^{4,5}$ For a given volume, a relative humidity value $(\mathrm{RH})$ could be assured by the effect of a saturated salt solution. A cylindrical closed chamber with a sealing lid was used, with a volume of 785 $\mathrm{mL}$. The selected salts were lithium chloride $(\mathrm{LiCl})$, magnesium chloride $\left(\mathrm{MgCl}_{2}\right)$, sodium chloride $(\mathrm{NaCl})$ and potassium sulfate $\left(\mathrm{K}_{2} \mathrm{SO}_{4}\right)$. The used quantities and expected relative humidity inside the chamber were:

Table S1: Salt solutions and expected relative humidity inside the closed chamber of a volume of $785 \mathrm{~mL}$.

\begin{tabular}{cccc}
\hline & Salt content $(\mathrm{g})$ & Water content $(\mathrm{ml})$ & Expected $\mathrm{RH} \%$ \\
\hline $\mathrm{LiCl}$ & 54.1 & 46.34 & $11 \%$ \\
$\mathrm{MgCl}_{2}$ & 58.25 & 20.46 & $33 \%$ \\
$\mathrm{NaCl}$ & 52.5 & 27.28 & $75 \%$ \\
$\mathrm{~K}_{2} \mathrm{SO}_{4}$ & 52.96 & 18.19 & $97 \%$ \\
\hline
\end{tabular}

The saturated salt solutions were prepared and poured inside in-house built containers, where a sample holder was placed to avoid the immersion of the functionalized silicon substrates. The chambers were tightly closed with a lid and relative humidity sensors (SEKSCC30-DB-Sensor, Sensirion) were used for the logging of the relative humidity and temperature inside of the chambers. The height of the polymer brushes was repeatedly measured after they were stored in the chambers for a certain period of time. The substrates were minimally rinsed with water and ethanol for 2 seconds, respectively, in order to removed the physisorbed brush which was degrafted on the surface. After that, the samples were dried with nitrogen stream and immediately after their height was measured by means of spectroscopic ellipsometry (SE). 
During degrafting experiments, the polymers were stored inside humid chambers where the constant humidity was provided by different salt solution. We assured that during storage the changes in the humidity were $<1 \%$. Larger variations occurred only during opening and closing of the chamber. We confirmed that the humidity reached its equilibrium value within 25 minutes (as depicted in Figure S2). The temperature in our facilities is kept constant at $22{ }^{\circ} \mathrm{C}$. We confirmed that the temperature in our chambers was indeed $22 \pm 0.2{ }^{\circ} \mathrm{C}$.

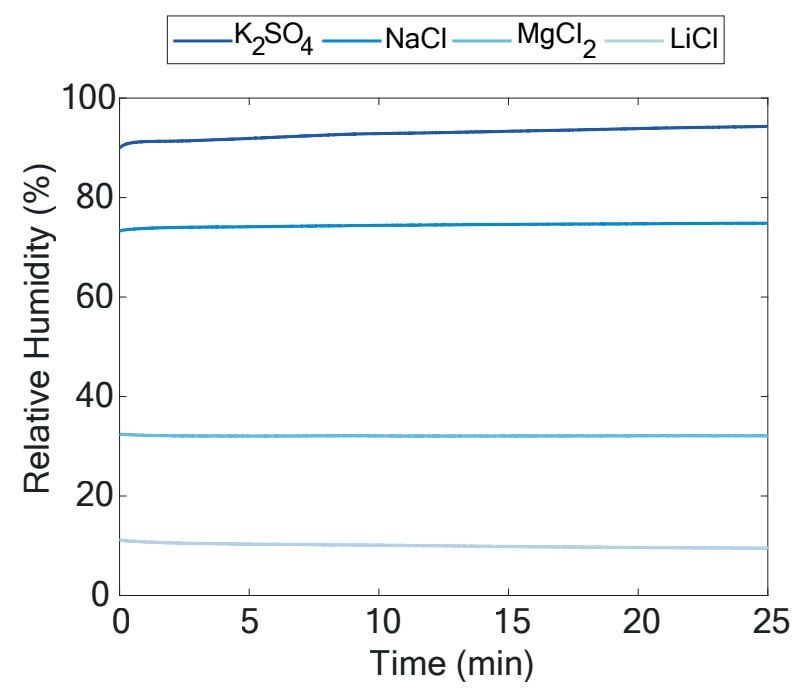

Figure S2: Stability measurements on relative humidities inside humid chambers over time.

\section{Characterization}

Static contact angle measurements were performed with the sessile drop technique, using an optical contact angle setup equipped with an electronic syringe unit (OCA15, Dataphysics, Germany). Fourier Transform Infrared spectroscopy (FTIR) spectra were obtained with an Alpha spectrometer (Bruker Scientific Instruments), by scanning the mid-infrared region (wavenumbers between 4000-400 $\mathrm{cm}^{-1}$. A piranha cleaned silicon substrate was scanned and used as background. ${ }^{1} \mathrm{H}-\mathrm{NMR}$ was conducted on a Bruker $400 \mathrm{MHz}$ instrument to obtain the monomer conversion and molecular weight $\left(M_{n}\right)$ estimation, using a different deuterated solvent for each polymer brush, as previously described. 
Ellipsometry measurements were performed using a M-2000X spectroscopic ellipsometer (J.A. Woollam) controlled by CompleteEASE software. The ellipsometric parameters $\Psi$ and $\Delta$ were recorded as a function of wavelength over the range of 245 to $1000 \mathrm{~nm}$. Heights of polymer brushes grown on silicon substrates were determined using the standard sample stage with 5 seconds sampling time at three angles of incidence $\left(65,70\right.$ and $\left.75^{\circ}\right)$. Calibration of the angle offset was performed with a silicon wafer coated with $25 \mathrm{~nm}$ of thermal oxide before each measurement day. Data analysis was performed with CompleteEASE software using an optical model comprised of the $\mathrm{Si}$ substrate, a $1 \mathrm{~nm}$ native $\mathrm{SiO}_{\mathrm{x}}$ layer and a Cauchy layer. The optical properties for the $\mathrm{Si}$ and $\mathrm{SiO}_{\mathrm{x}}$ layers were taken from the software material database. The polymer brush films were fit to a second order Cauchy relation $\left(A_{\mathrm{n}}\right.$ and $\left.B_{\mathrm{n}}\right)$. Since the C parameter did not yield improved fitting results, it was set to 0 . The polymer brush film was considered to be uniform, and the roughness of the polymeric layers was taken into account by obtaining spectra from 3 different points of the sample. However, the differences in thickness were minimal so a gradient model was not needed. The availability of the ellipsometry setup was limited and hence, the intervals of degrafting experiments were not equidistant.

AFM measurements were performed in tapping mode, using a Bruker system on a MultiMode 8 with a NanoScope V controller a JV vertical engage scanner and a stainless steel probe holder with integrated piezo oscillates control (Bruker Scientific Instruments, Billerica, Massachusetts, USA). NanoScope Analysis was used to process the images and obtain step height values at varying relative humidity. The relative humidity inside the chamber was controlled by adjusting the relative flow rates of a dried nitrogen stream and a watersaturated nitrogen stream, as represented in Figure S9. A general supply of dry nitrogen was separated into two streams. The first one was passed with tubing via a control valve an was subsequently connected to two bubblers filled with milliQ water. The temperature of the water inside the bubblers was controlled by placing them in a water bath and was kept at 
$25^{\circ} \mathrm{C}$. The nitrogen stream containing water vapor passed through the bubbles and was then connected to the AFM chamber. The second dry nitrogen stream was directly connected to the AFM chamber, controlling its flow with a valve. The relative humidity inside the chamber fluctuated by changing the flow of the dry and water vapoured nitrogen streams with the valves on each stream. Inside the chamber, the relative humidity was monitored using a relative humidity sensor (Sensirion SEK-SCC30-DB-Sensor). For safety reasons, in order to avoid any possible condensation of water in the surrounding of the AFM scanner, we decided to limit the relative humidity to $80 \%$. Silicon substrates coated with polymer brushes were gently scratched with a Teflon tweezer in order to expose the underlying silicon prior to AFM measurements. The substrates were then rinsed to remove the residual brush coating and this way, the brush thickness profiles were obtained.

\section{Supporting results}

The change of surface wettability after different surface functionalizations is depicted in table S2 and Figure S3. Results show higher contact angle values for hydrophobic polymer brushes, and low values for the hydrophilic ones. The measured contact angles are in agreement with literature values. ${ }^{1-3,6}$

Table S2: Obtained contact angle values of silicon substrates coated with different functional layers.

\begin{tabular}{cc}
\hline Sample content & Static contact angle $\left( \pm 5^{\circ}\right)$ \\
\hline \hline Clean silicon & $<10$ \\
Si-APTES & 47 \\
Si-APTES-BiBB & 71 \\
PMMA & 64 \\
PHEMA & 50 \\
PSPMA & 10 \\
PMPC & 16 \\
\hline
\end{tabular}



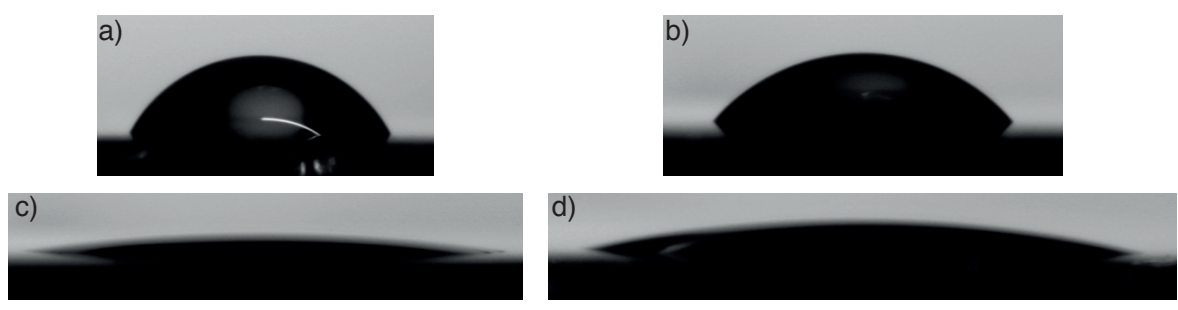

Figure S3: Water droplets of $2 \mu \mathrm{L}$ used for static contact angle measurements on silicon substrates functionalized with polymer brushes, being a) PMMA, b) PHEMA, c) PSPMA and d) PMPC polymer brushes.

The obtained FTIR spectra of the four studied polymer brushes are discussed in the following section. The location of the peaks obtained for each polymer brush is in agreement with previously reported results. ${ }^{7-10}$ For PMMA ( Figure S4 (a)), the corresponding peaks were found at: $1260-1040 \mathrm{~cm}^{-1}(\mathrm{C}-\mathrm{O}-\mathrm{C}$ stretching vibration $), 1450 \mathrm{~cm}^{-1}\left(\mathrm{CH}_{3}\right.$ deformation vibration), $1730 \mathrm{~cm}^{-1}$ ( $\mathrm{C}=\mathrm{O}$ ester bond stretching vibration). Figure $\mathrm{S} 4$ (b) shows the FTIR spectrum of PHEMA brushes with its corresponding characteristic peaks, being: $1730 \mathrm{~cm}^{-1}(\mathrm{C}=\mathrm{O}$ ester bond stretching vibration $), 1260-1040 \mathrm{~cm}^{-1}(\mathrm{C}-\mathrm{O}-\mathrm{C}$ stretching vibration) and $1450 \mathrm{~cm}^{-1}\left(\mathrm{CH}_{3}\right.$ deformation vibration). Figure S4 (c) shows the FTIR spectrum of PSPMA brushes with its corresponding characteristic peaks, being: $1450 \mathrm{~cm}^{-1}\left(\mathrm{CH}_{3}\right.$ deformation vibration), $1730 \mathrm{~cm}^{-1}\left(\mathrm{C}=\mathrm{O}\right.$ ester bond stretching vibration) and $1190 \mathrm{~cm}^{-1}$ and $1042 \mathrm{~cm}^{-1}\left(\mathrm{SO}_{3}^{-}\right.$symmetric and asymmetric stretching). Lastly, Figure $\mathrm{S} 4(\mathrm{~d})$ shows the FTIR spectrum of PMPC brushes with its corresponding characteristic peaks, being: 1730 $\mathrm{cm}^{-1}(\mathrm{C}=\mathrm{O}$ ester bond stretching vibration $) 1235 \mathrm{~cm}^{-1}(\mathrm{O}=\mathrm{P}-\mathrm{O}$ group asymmetric stretching), $1089 \mathrm{~cm}^{-1}\left(\mathrm{C}-\mathrm{N}^{+}-\mathrm{C}\right.$ group stretching) and $962 \mathrm{~cm}^{-1}$ ( $\mathrm{P}-\mathrm{O}$ group bending). 

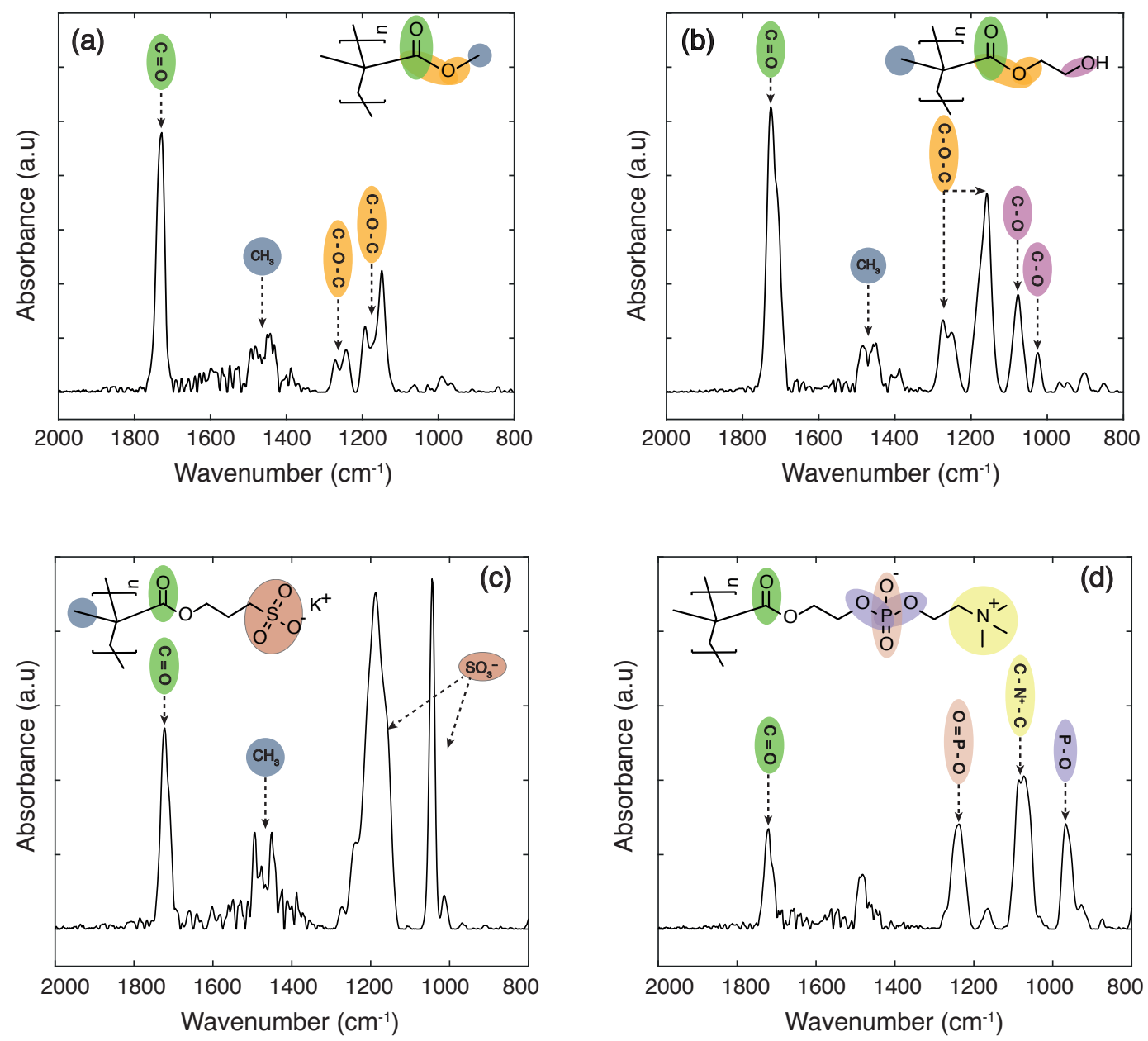

Figure S4: FTIR spectrum of a) PMMA, b) PHEMA, c) PSPMA and d) PMPC polymer brushes grown on silicon substrates. Each figure includes the chemical side group which belongs to each of the selected IR bands.

We monitored the monomer consumption over time, which is translated to the growth of polymers. In these experiments, we quantify the decrease on the intensity and/or location of the chemical shift peaks quantified by ${ }^{1} \mathrm{H}-\mathrm{NMR}$ belonging to the monomers, which allows us to calculate its consumption and thereby, the reactions conversion. The molecular weight is calculated by following equation 1 :

$$
M_{n}=\left([M]_{0} /[R X]_{0}\right) \cdot \operatorname{Conv} \cdot M_{w}(M)
$$


Where $[M]_{0}$ is the initial monomer concentration, $[R X]_{0}$ is the initial concentration of sacrificial initiator, Conv is the conversion, calculating by difference in the intensity of the characteristic monomer peak(s) and $M_{w}(M)$ is the weight-averaged molecular weight of the monomer.
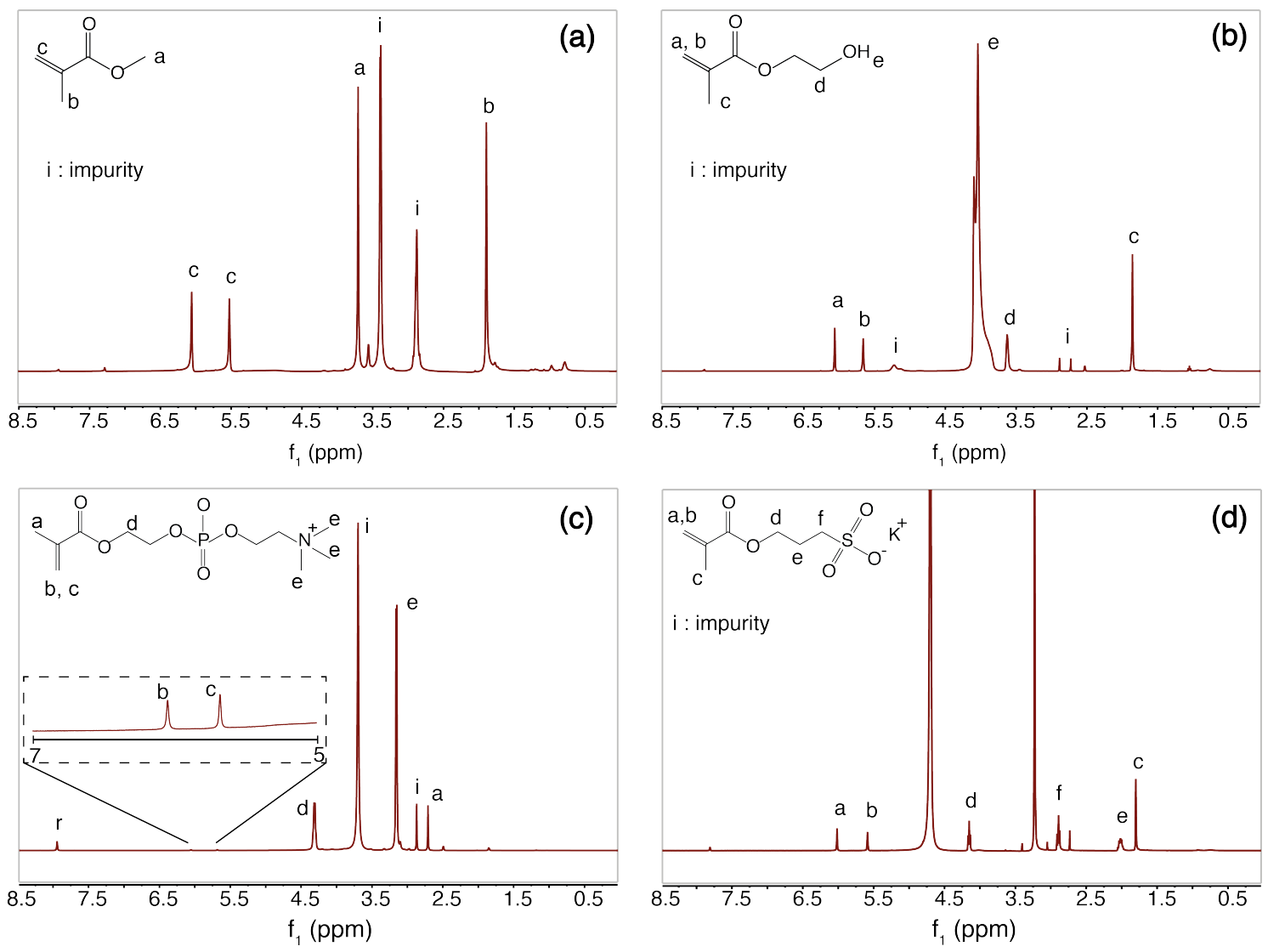

Figure S5: ${ }^{1} \mathrm{H}-\mathrm{NMR}$ spectra of a) PMMA in chloroform-d, b) PHEMA in DMSO- $\mathrm{d}_{6}$, c) PMPC in $\mathrm{D}_{2} \mathrm{O}$ d) PSPMA in $\mathrm{D}_{2} \mathrm{O}$, all initiated by sacrificial ethyl $\alpha$-bromoisobutyrate. 
Table S3: Estimated values of the grafting densities of different polymer brushes, grafted on silicon substrates via APTES anchors. The obtained molecular weight by means of ${ }^{1} \mathrm{H}-\mathrm{NMR}$ spectra is reported.

\begin{tabular}{cccc}
\hline Polymer brush & $\sigma\left(\right.$ chains $\left./ \mathrm{nm}^{2}\right)$ & $M_{\mathrm{n}}(\mathrm{KDa})$ & Thickness $(\mathrm{nm})$ \\
\hline \hline PMMA & 0.42 & 10 & $25.5 \pm 0.8$ \\
PHEMA & 0.60 & 65 & $96.2 \pm 0.2$ \\
PSPMA & 0.50 & 42 & $78.1 \pm 0.8$ \\
PMPC & 0.65 & 15 & $59.5 \pm 0.5$ \\
\hline
\end{tabular}
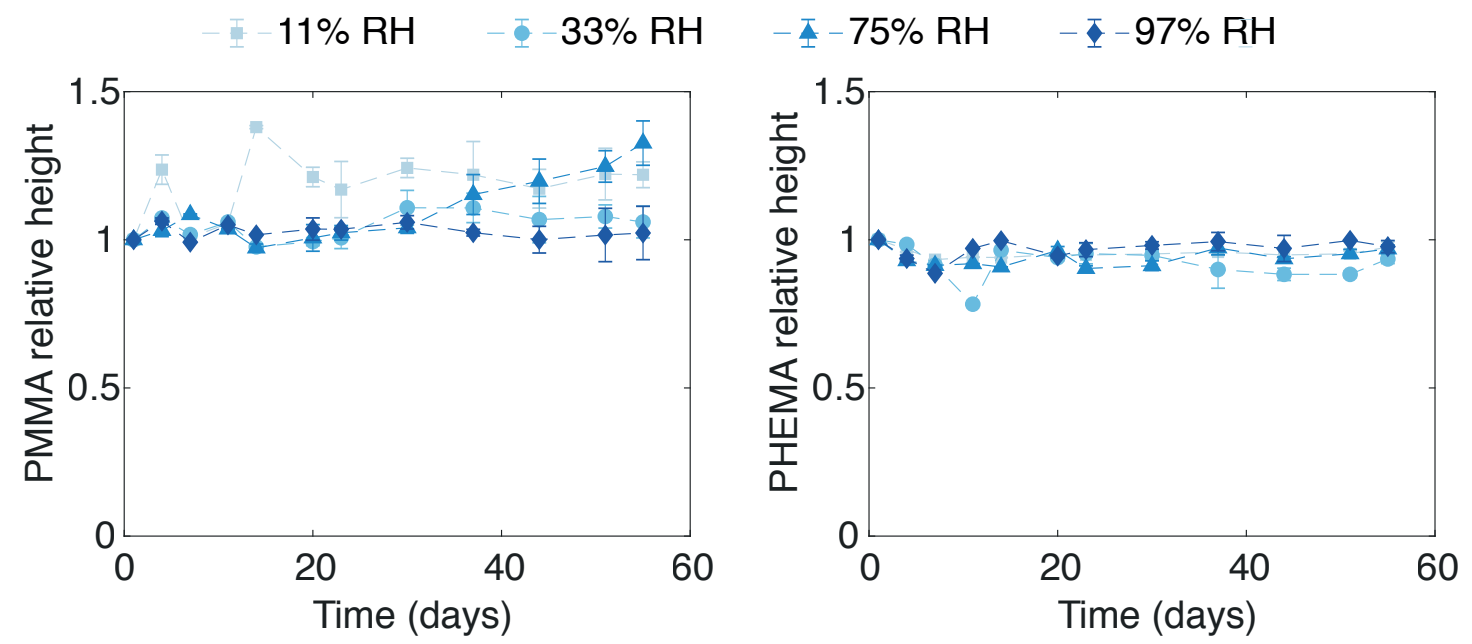

Figure S6: Degrafting of PMMA (left) and PHEMA (right) polymer brushes in humid air of varying relative humidities, being from lighter to dark blue: $11 \% \mathrm{RH}, 33 \% \mathrm{RH}, 75 \% \mathrm{RH}$ and $97 \% \mathrm{RH}$, respectively. The initial height of the PMMA brushes was $25 \mathrm{~nm}$, with $\sigma=0.42$ chains $/ \mathrm{nm}^{2}$. For PHEMA brushes, the initial height was $96 \mathrm{~nm}$, with $\sigma=0.60$ chains $/ \mathrm{nm}^{2}$. 

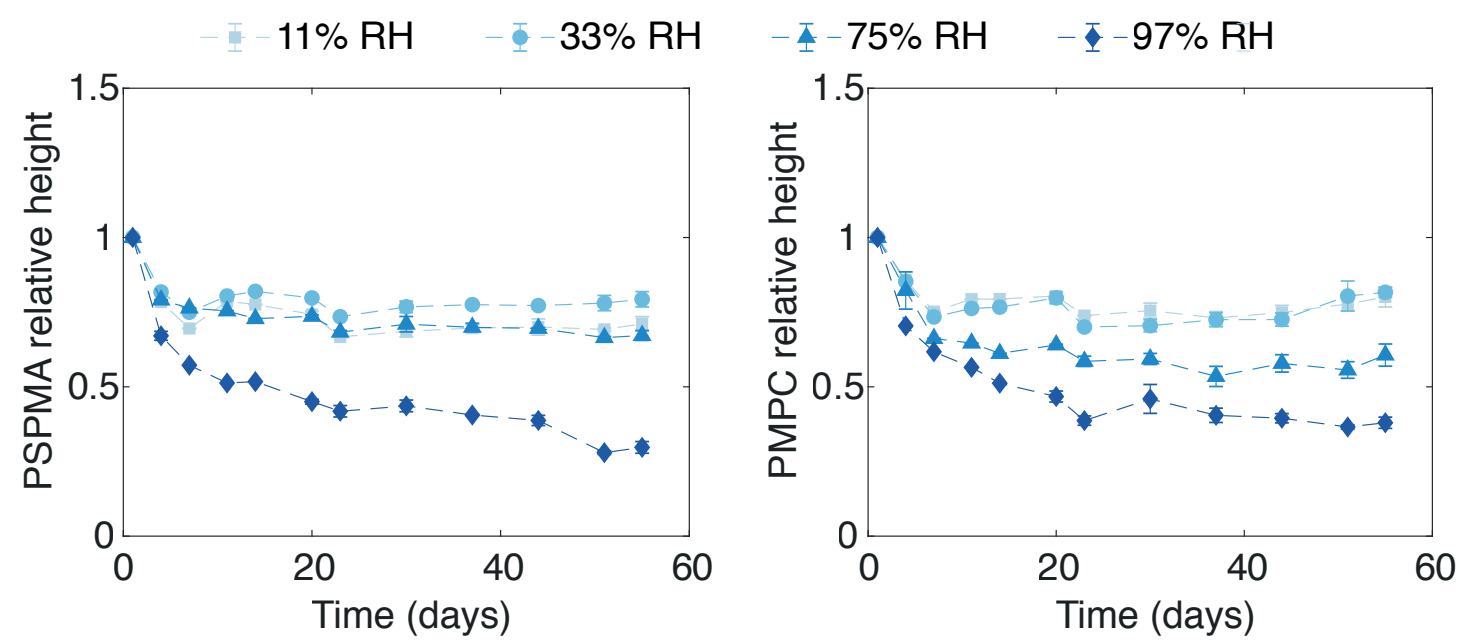

Figure S7: Degrafting of PSPMA (left) and PMPC (right) polymer brushes in humid air of varying relative humidities, being from lighter to dark blue: $11 \% \mathrm{RH}, 33 \% \mathrm{RH}, 75 \% \mathrm{RH}$ and $97 \% \mathrm{RH}$, respectively. The initial height of the PMPC brushes was $60 \mathrm{~nm}$, with $\sigma=0.65$ chains $/ \mathrm{nm}^{2}$. For PSPMA brushes, the initial height was $78 \mathrm{~nm}$, with $\sigma=0.50$ chains $/ \mathrm{nm}^{2}$.

Assuming that the polymers degraft from the surface at their anchor points, we can estimate the grafting density $(\sigma)$ by means of Equation 2 :

$$
\sigma=\rho \cdot N_{A} \cdot d / M_{n}
$$

Where $\rho$ is the bulk density of the brush, $N_{A}$ is Avogadro's number, $d$ is the thickness of the film and $M_{n}$ the estimated molecular weight by means of ${ }^{1} \mathrm{H}-\mathrm{NMR}$. Via this calculation, we obtain figures which have the same shape as the degrafting trends shown in Figures S6 and S7, having this time the grafting density as their Y axes: 

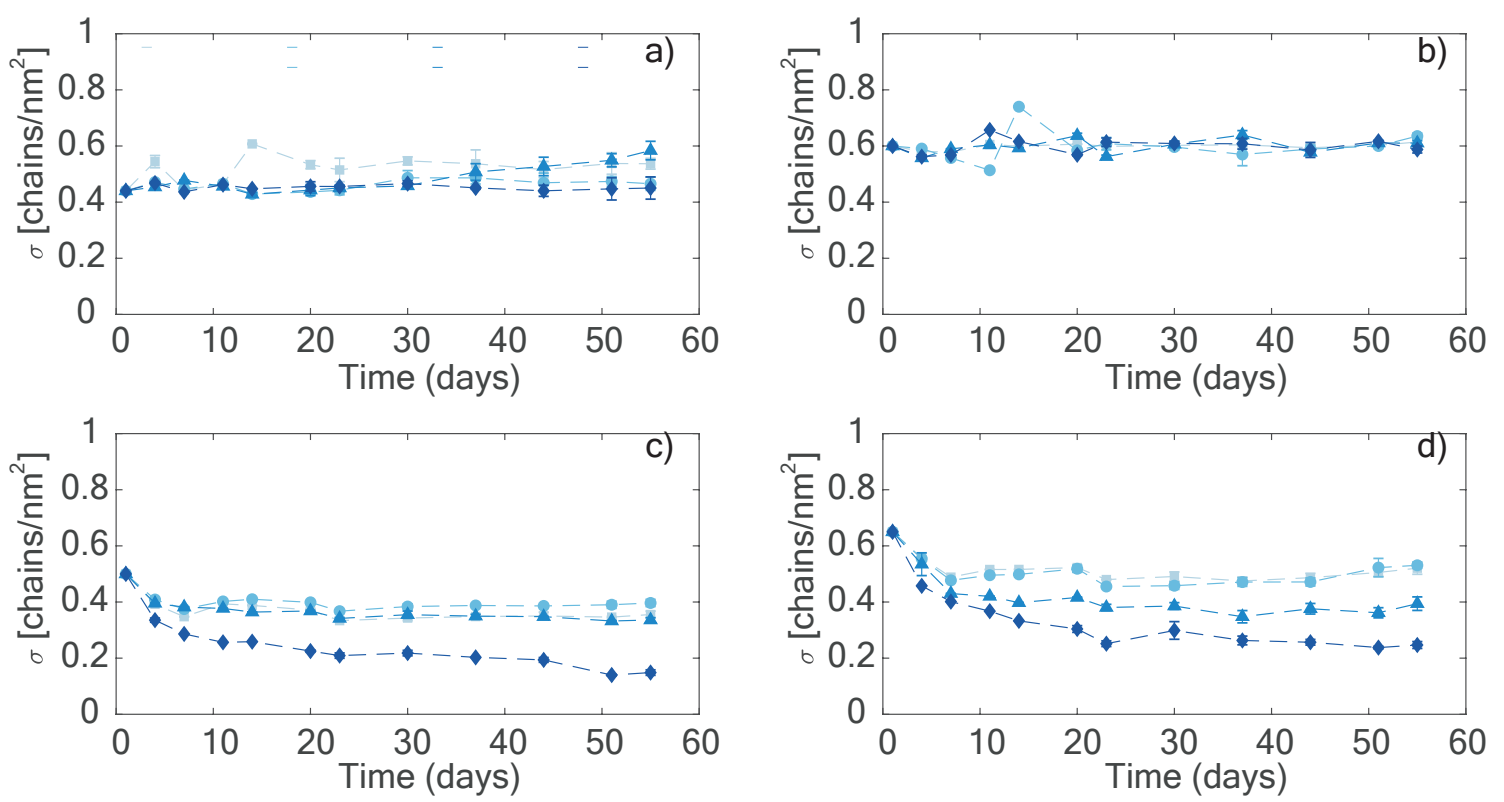

Figure S8: Variations of grafting density of tethered polymer brushes on silicon substrates over time, being a) PMMA, b) PHEMA, c) PSPMA and d) PMPC brushes. The color scheme indicates the relative humidity inside the chambers where the brushes were stored, being from lighter to darker blue:: 11\% RH, 33\% RH, $75 \% \mathrm{RH}$ and 97\% RH, respectively.

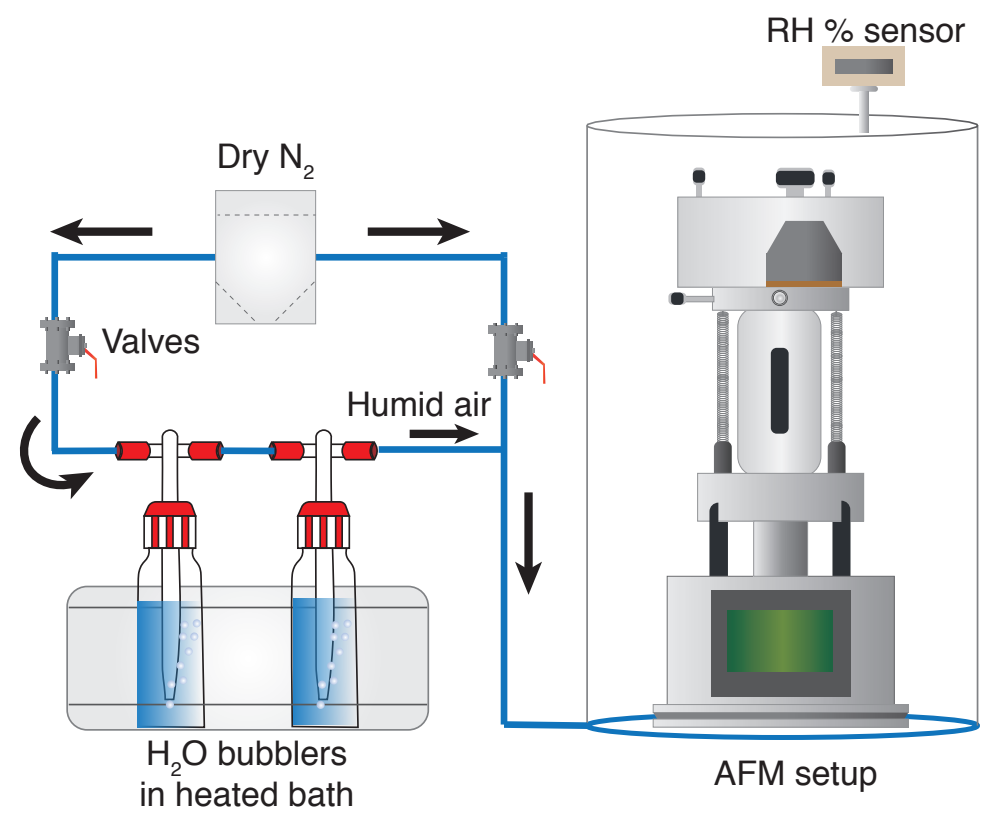

Figure S9: Schematic representation of the implemented humidity setup for AFM measurements at different relative humidities. 


\section{References}

(1) Tas, S.; Kopec, M.; van der Pol, R.; Cirelli, M.; de Vries, I.; Bölükbas, D. A.; Tempelman, K.; Benes, N. E.; Hempenius, M. A.; Vancso, G. J., et al. Chain EndFunctionalized Polymer Brushes with Switchable Fluorescence Response. Macromol. Chem. Phys. 2019, 220, 1800537.

(2) Yu, Y.; Vancso, G. J.; de Beer, S. Substantially enhanced stability against degrafting of zwitterionic PMPC brushes by utilizing PGMA-linked initiators. Eur. Polym. J. 2017, 89, 221-229.

(3) Benetti, E. Molecular engineering of designer surfaces by controlled radical polymerizations: brushes, hedges and hybrid grafts. Ph.D. thesis, University of Twente, Netherlands, 2009.

(4) Quincot, G.; Azenha, M.; Barros, J.; Faria, R. Use of salt solutions for assuring constant relative humidity conditions in contained environments. Fundação para a Ciência e a Tecnologia 2011,

(5) Greenspan, L., et al. Humidity fixed points of binary saturated aqueous solutions. $J$. Res. Natl. Bur. Stand. 1977, 81, 89-96.

(6) Yu, Y.; Cirelli, M.; Li, P.; Ding, Z.; Yin, Y.; Yuan, Y.; De Beer, S.; Vancso, G. J.; Zhang, S. Enhanced Stability of Poly (3-sulfopropyl methacrylate potassium) Brushes Coated on Artificial Implants in Combatting Bacterial Infections. Ind. Eng. Chem. Res. 2019, 58, 21459-21465.

(7) Ramesh, S.; Leen, K. H.; Kumutha, K.; Arof, A. FTIR studies of PVC/PMMA blend based polymer electrolytes. Spectrochim. Acta, Part A 2007, 66, 1237-1242.

(8) Passos, M. F.; Fernández-Gutiérrez, M.; Vázquez-Lasa, B.; San Román, J.; Maciel Filho, R. PHEMA-PLLA semi-interpenetrating polymer networks: A study of their 
swelling kinetics, mechanical properties and cellular behavior. Eur. Polym. J. 2016, 85, $150-163$.

(9) Patel, R.; Chi, W. S.; Ahn, S. H.; Park, C. H.; Lee, H.-K.; Kim, J. H. Synthesis of poly (vinyl chloride)-g-poly (3-sulfopropyl methacrylate) graft copolymers and their use in pressure retarded osmosis (PRO) membranes. Chem. Eng. J. 2014, 247, 1-8.

(10) Moro, T.; Takatori, Y.; Kyomoto, M.; Ishihara, K.; Saiga, K.-i.; Nakamura, K.; Kawaguchi, H. Surface grafting of biocompatible phospholipid polymer MPC provides wear resistance of tibial polyethylene insert in artificial knee joints. Osteoarthritis Cartilage 2010, 18, 1174-1182. 